\title{
The Large Magellanic Cloud with the Cherenkov Telescope Array
}

\author{
María Isabel Bernardos ${ }^{1, a}$, María Benito ${ }^{2}$, Fabio locco $^{2}$, Salvatore Mangano ${ }^{1}$, Olga Sergijenko ${ }^{4}$, \\ Ekaterina Karukes ${ }^{2}$, and Lili Yang ${ }^{3}$ for the CTA Consortium \\ ${ }^{1}$ Centro de Investigaciones Energéticas, Medioambientales y Tecnológicas \\ ${ }^{2}$ ICTP-South American Institute for Fundamental Research. Instituto de Física Teórica - UNESP. \\ ${ }^{3}$ University of Nova Gorica \\ ${ }^{4}$ National Academy of Sciences of Ukraine, Kiev, Ukraine
}

\begin{abstract}
The Large Magellanic Cloud (LMC) is a spiral galaxy, satellite of the Milky way with a high star formation activity. It represents a unique laboratory for studying an extended and spatially resolved star-forming galaxy through gamma-ray observatories. Therefore, the LMC survey is one of the key science projects for the Cherenkov Telescope Array (CTA), the next-generation ground-based gamma-ray observatory. In this document we present the work performed over the last year by the CTA working group dedicated to the LMC, in order to offer a first characterization of the LMC at TeV energies. We have performed detectability forecasts based on the expected CTA performance for all sources in the region of interest of the LMC with known emission at GeV energies and above. Based on previous observations made by Fermi-LAT and H.E.S.S. we have characterized all point sources, extended sources and diffuse emission produced by cosmic-ray propagation, extrapolating their spectra to CTA energies. Finally, we have characterized the signal expected by different annihilation mechanisms of dark matter (DM) particles within the LMC, computing the detection sensitivity curve for this target in the cross-section-to-mass plane.
\end{abstract}

\section{Introduction}

The LMC is a satellite galaxy of the Milky Way with unique characteristics that make it a valuable target for gamma-ray observations. It is located in the Southern hemisphere at a galactic latitude of $-32.9^{\circ}$ off the galactic plane, with a disk shape almost faced on (with a small inclination angle of 30$40^{\circ}$ ). The LMC is one of the nearest active star-forming galaxies, at a distance of about $50 \mathrm{kpc}$ (with an uncertainty of $2 \%$ [1]. In this work, we study the forecasts on detecting the already known gammaray emission coming from sources in LMC with the Southern Array of CTA, the next-generation of ground-based gamma-ray observatories which is already in construction. We will cast simulations of a survey of the LMC, based on an emission model built from catalogs of present gamma-ray telescopes. In section 2 We talk about the previous gamma-ray observations of the LMC performed by FermiLAT and H.E.S.S. In section 3 the analysis procedure is described. A study on the sensitivity of CTA South for the detection of a DM annihilation gamma-ray signal coming from the LMC in presented in section 4.

\footnotetext{
a e-mail: mariaisabel.bernardos@ ciemat.es
} 


\section{Previous observations of the LMC in gamma-ray}

The LMC has been already observed in the gamma ray by Fermi-LAT and H.E.S.S., with the identification of several gamma-ray sources of different nature: Supernova Remnant 132D, the Pulsar Wind Nebula 157B, the very interesting superbubble 30 Doradus C [2] and the gamma-ray binary J0534.16732/HESS J0536-675, whose period was recently measured [3]. Also, H.E.S.S. acquired more than $200 \mathrm{~h}$ of data pointing at the position where the Supernova (SN) 1987A exploded in the last century, but they did not detect any gamma-ray emission [4]. However, they calculated upper limits on the flux of SN1987A which fall far above the sensitivity of CTA South (for $50 \mathrm{~h}$ of observations), making it a key target for CTA.

\section{Forecasts on detection of known gamma-ray sources}

We aimed to test the capability of CTA to detect and study the already known gamma-ray emission from the LMC. To do so, we have retrieved information of the gamma-ray sources detected in the LMC by other experiments to build an emission model. We have used one of the proposed analysis softwares for CTA, the ctools [5] combined with a custom pipeline to do a likelihood fit over a simulated survey of the LMC, and we have calculated the significances of the individual sources as seen with CTA.

\subsection{Emission model of the LMC}

We have retrieved a list of gamma-ray sources detected in the surroundings of the LMC, a Region of Interest (ROI) of $10^{\circ}$ radius using the 3FGL [6] and the 3FHL [7] catalogs from Fermi-LAT, the Fermi collaboration paper [8] and H.E.S.S. papers [2],[3]. We have combined the data of the two experiments and have extrapolated the spectra of the sources to CTA energies (up to $100 \mathrm{TeV}$ ). Our LMC emission model has three main components: 10 point sources, 3 extended sources and a diffuse emission produced by cosmic-ray interaction with the interstellar medium. For the latter we have used models for leptonic and hadronic emission components (courtesy of Pierrick Martin [9]). A skymap of the full emission model is shown in Figure 1.

\subsection{Simulations and analysis: Likelihood fitting}

We have simulated $340 \mathrm{~h}$ of observation of the LMC divided in 6 pointings ( $56.6 \mathrm{~h}$ each) surrounding the LMC center(see Figure 1). Then we have performed a binned analysis with a spatial resolution of $0.1^{\circ}$ per pixel, and 20 energy bins from $100 \mathrm{GeV}$ to $100 \mathrm{TeV}$. We have used the Prod $3 \mathrm{~b}$ calibration function South_z40_average_50h. With the tool ctmodel we have built a model of predicted number counts of the observations (to be used as mock data) and for each indiviual source in the emission model (to build a model from the combination of all sources). We performed a binned likelihood analysis using a simple Poisson likelihood function:

$$
L=\prod_{i}^{N} \frac{m_{i}^{n_{i}}}{n_{i} !} e^{-m_{i}}
$$

In Equation 1, $N$ is the total number of bins (energy and spatial bins), $n_{i}$ is the number of data counts (photons) in the $i$-th bin, and $m_{i}$ is the estimated number of counts in the $i$-th bin by the model. In the fit, the free parameters are the normalizations of the spectral models for the different sources. We defined the significance of a source in terms of the test statistics (TS)(see Equation 2), where L is the maximum likelihood, and $L_{\text {null }}$ is the Likelihood of the null hypothesis for that specific source. 

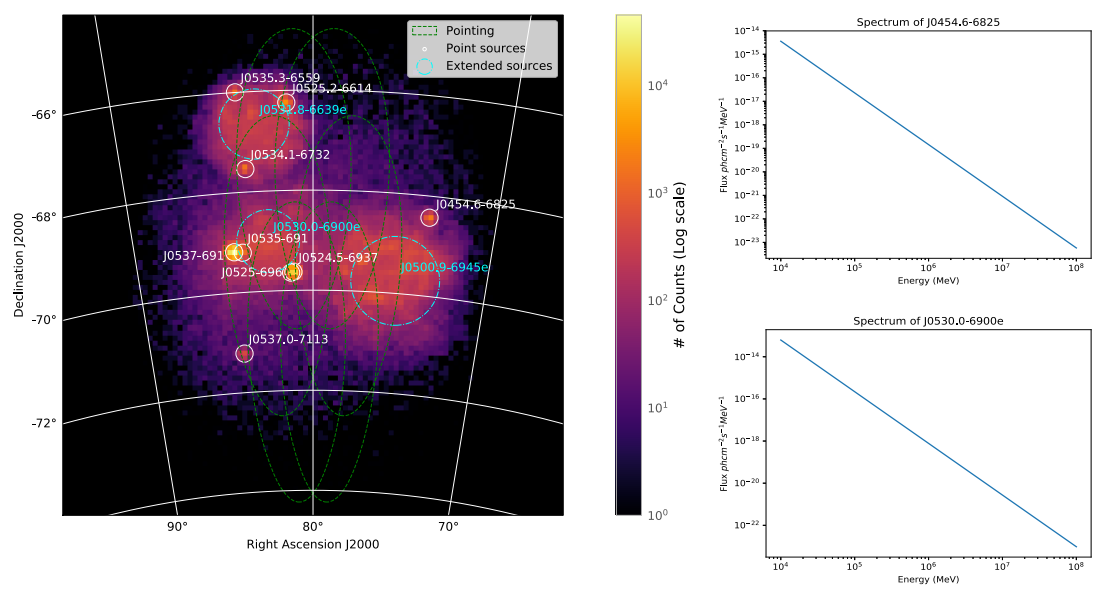

Figure 1: Left:Skymap of the LMC simulated in this work obtained with ctobssim and ctskymap tools. Green dashed lines represent the 6 pointings of the LMC survey. White circles mark the position of point sources and cyan point-dashed circles mark the position of the three extended sources. The atmospheric cosmic-ray background has been substracted in this image for a better appreciation of the structure details. Top right: Spectrum of one of the point sources, J0454-6825. Bottom right: Integrated spectrum of the diffuse source J0530-6900e

$$
T S=2 \log \frac{L}{L_{\text {null }}}
$$

\subsection{Significances and Correlations}

The results of the likelihood fit are listed in Table 1 with the significances for the sources. We also have studied the correlations between extended sources, which can affect their significances as photons from one source can be assigned to another one with which the former has a high correlation. In particular, we have noticed a considerable anti-correlation (correlation coefficient $r \approx-0.5$ ) between the leptonic emission model and the extended source J0530.0-6900e. We can do a temptative explanation of this correlation knowing that the source is identified as the west region of the star-forming region 30 Doradus, whose activity can be enhancing the leptonic diffuse emission produced by electrons inverse-compton scattering the radiation field, that experience very strong losses and don't travel very far form their sources [10].

\section{Indirect detection of Dark Matter annihilation in the LMC with CTA}

The LMC can be an interesting source for indirect DM searches. Having an astrophysical factor for DM annihilation $J 10^{20} \mathrm{GeV}^{2} \mathrm{~cm}^{-} 5$ [11] and being an extended object, we can use the spatial structure of its baryonic emission to unveil a possible diffuse DM signal coming from its halo. We have studied the sensitivity of CTA to detect a signal from different DM models depending on DM particle mass, annihilation channel and density profile. We have calculated 95\% CL limits on the DM annihilation cross section through a maximum-likelihood analysis over 300 realizations of the LMC data. As a result, the sensitivity curve with $95 \%$ and $\% 84$ containment bands (same bands used by [11]) expected 
Table 1: Significance of the astrophysical sources considered in the ROI of the LMC for this work

\begin{tabular}{ll}
\hline Source name & Significance $(\sigma)$ \\
\hline J0500.9-6945e & 88.41 \\
J0530.0-6900e & 19.98 \\
J0531.8-6639e & 111.97 \\
J0537-691 & 264.43 \\
J0524.5-6937 & 116.62 \\
J0534.1-6732 & 44.58 \\
J0525.2-6614 & 83.40 \\
J0535.3-6559 & 64.72 \\
J0454.6-6825 & 47.81 \\
J0537.0-7113 & 10.66 \\
J0535-691 & 21.05 \\
J0525-696 & 40.58 \\
J0509.9-6418 & 0.50 \\
\hline
\end{tabular}

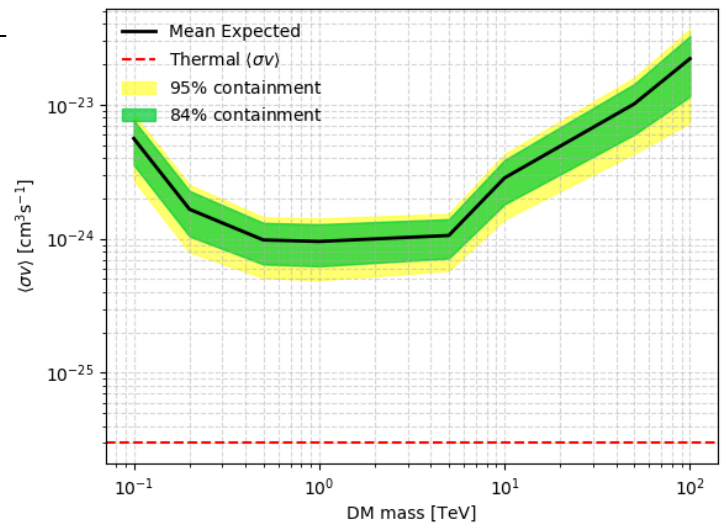

Figure 2: Sensitivity curve for $W^{+} W^{-}$annihilation channel, using a NFW density profile for the LMC DM halo.

for the $W^{+} W^{-}$annihilation channel with a Navarro-Frenk-White (NFW) density profile is shown in Figure 2. A visual inspection reveals that the sensitivity curve is several orders of magnitude above the thermal cross section, compatible with the results for DM searches in the LMC from [11]. This preliminary result confirms that the LMC is not a prime target for searches of signal of annihilating Weakly Interacting Massive Particles (WIMPs) DM.

\section{Acknowledgements}

We gratefully acknowledge financial support from the agencies and organizations listed here: http: //www.cta-observatory.org/consortium_acknowledgments. This research has made use of the CTA instrument response functions provided by the CTA Consortium and Observatory, see https://www.ctaobservatory.org/science/cta-performance/ (version prod3b-v1) for more details.

\section{References}

[1] G.e.a. Pietrzyński, 495, 76 (2013), 1303.2063

[2] A. Abramowski et al. (H.E.S.S.), Science 347, 406 (2015), 1501.06578

[3] N. Komin, M. Haupt (HESS) (2017), 1708.03171

[4] N.e.a.f. Komin, ArXiv e-prints (2012), 1201.0639

[5] J.e.a. Knödlseder, "Astron. Astrophys." 593, A1 (2016), 1606.00393

[6] F. Acero et al. (Fermi-LAT), Astrophys. J. Suppl. 218, 23 (2015), 1501.02003

[7] M. Ajello et al. (Fermi-LAT) (2017), 1702.00664

[8] M. Ackermann et al. (Fermi-LAT), Astron. Astrophys. 586, A71 (2016), 1509.06903

[9] Cherenkov Telescope Array Consortium, ArXiv e-prints (2017), 1709.07997

[10] P. Martin, Astron. Astrophys. 564, A61 (2014), 1402.0383

[11] M.R.e.a. Buckley, Phys. Rev. D91, 102001 (2015), 1502.01020 